Es gibt Zufälle, die sind so zufällig, dass es sich schwerlich nur um Zufall handeln kann.

\title{
Zwei gestempelte Silberbarren und ein spätantiker Goldbarren aus Crasna (Rumänien)
}

\author{
Rainer Wiegels
}

Im Jahr 2013 erhielten wir Kenntnis von zwei unabhängig voneinander erworbenen gestempelten Silberbarren, deren genaue Herkunft unbekannt ist, die jedoch aus dem südöstlichen Europa stammen sollen, was durchaus plausibel erscheint. ${ }^{1}$ Beide Barren wurden von uns 2014 in einer eigenen kleinen, von der Degussa Goldhandel GmbH (München) herausgegebenen Schrift veröffentlicht. ${ }^{2}$

Bei dem von uns an genannter Stelle unter Nr. 1 behandelten Silberbarren ${ }^{3}$ sind Form und Stempelung auffällig, denn die auf dem schmalen, flachen Silberbarren eingeprägten Stempel sind gut bekannt von spätantiken Goldbarren (!) aus Crasna (com. Sita Buzăului, jud. Covasna, Rumänien), ein Waldareal, welches zur Zeit der Entdeckung eines Fundkomplexes mehrerer Goldbarren im Jahr 1887 zu Ungarn gehörte, aber der rumänischen Grenze unmittelbar benachbart lag. ${ }^{4}$ Die schmal-längliche Form in der Art einer flachen Stange (Abb. 1) ist für gestempelte Silberbarren bislang nicht bekannt, sie entspricht aber sehr wohl - zumindest im Grundsätzlichen - derjenigen von Goldbarren, die allerdings länger sind und auch als „Siegellackstangen“ bezeichnet wurden. ${ }^{5}$

Eine ausführliche Wiederholung unserer Interpretation des Fundes erübrigt sich an dieser Stelle. Im Folgenden sei nur das in unserem Zusammenhang Notwendige rekapituliert: Neben den erwähnten Auffälligkeiten haben uns insbesondere die Metallanalyse und die Stempelinhalte zu dem Schluss geführt, dass der Barren als solcher nicht antik sein kann, wohl aber irgendwie im Kontext der genannten Goldbarren stehen muss. Hauptgründe gegen eine antike Zeitstellung waren zum einen der reine Silbergehalt des Barrens ohne die geringsten Spuren von Blei oder Gold, was bei Schmelzvorgängen in der Antike nicht möglich war und erst in der Neuzeit gelang; zum anderen die in der Antike ausschließlich auf reines Gold bezügliche Abkürzung obryzum (aurum) bzw. obryza im Textfeld des mittleren von drei Stempeln auf diesem Barren, womit der Reinheitsgehalt angezeigt bzw. bestätigt wurde. ${ }^{6}$ Bei Silber wurde dieses mit pusulatum (argentum) mit verschiedenen Abkürzungsformen garan-

\footnotetext{
${ }^{1}$ Als mögliche, aber ungesicherte Herkunft für das im Folgende besprochene Exemplar wurde Ungarn genannt.

${ }^{2}$ R. WIEGELS, Zwei gestempelte Silberbarren und die spätantiken Goldbarren aus Siebenbürgen (München 2014). - Kenntnis von den Barren erhielten wir von R. Eberlein, Degussa Goldhandel GmbH, in deren Sammlung die beiden Objekte jetzt verwahrt werden. Für seine Auskünfte und Hilfestellungen sind wir Herrn Eberlein zu Dank verpflichtet.

${ }^{3}$ Der dort unter Nr. 2 behandelte Silberbarren spielt für das Folgende keine Rolle.

${ }^{4}$ Hierzu und mit den entsprechenden Verweisen und umfassenden Literaturangaben WIEGELS (Anm. 2) passim, bes. 13 Anm. 7. - Im Folgenden wird auf eine ausführliche Wiedergabe der einschlägigen Literatur verzichtet. S. dazu das Verzeichnis bei WIEGELS a. O. 90-95 oder R. WIEGELS, Silberbarren der römischen Kaiserzeit. Katalog und Versuch einer Deutung. Freiburger Beiträge zur Archäologie und Geschichte des ersten Jahrtausends 7 (Rahden 2003) 133-172.

${ }^{5}$ Vgl. hierzu den Katalog bei WIEGELS (Anm. 2) 42 ff. mit den entsprechenden Abbildungen.

${ }^{6}$ Diskussion und Belege bei WiEgEls (Anm. 2) 22 ff., bes. 22 Anm. 26.
} 
tiert. Unabhängig davon sei aber schon an dieser Stelle notiert, dass bei Gold mittels Stempelung die Garantie des Reinheitsgehaltes, bei Silber aber das Gewicht von erstrangiger Bedeutung war.

Man könnte es bei der schlichten Feststellung einer Fälschung bewenden lassen und zur Tagesordnung übergehen, wenn nicht ein überraschender, aktueller, Neufund' zur erneuten Beschäftigung mit diesem und unserem vorgenannten Barren raten würde, bei der einige Vertiefungen und Akzentuierungen in Bezug auf die Argumentation die Folge sind.

In einem Londoner Auktionskatalog zum März 2015 wird bzw. wurde ein gestempelter Silberbarren zur Versteigerung angeboten unter der head line: „Very Rare and Interesting Roman Silver Ingot" und auch dort mit Vor- und Rückseite abgebildet (hier Abb. 2). ${ }^{7}$ In der beigefügten Erläuterung wird der Barren ins späte 4. Jahrhundert datiert und die Stempelung ebenfalls mit denjenigen der Goldbarren aus früher - Kronstadt, heute rumänisch Braşov (ungarisch Brasso), verglichen. ${ }^{8}$ Dazu wird auf einschlägige angelsächsische Literatur verwiesen mit Abbildung zweier im British Museum in London befindlicher Barren (in einem Fall das Fragment eines solchen) aus dem Fundkomplex von Crasna. ${ }^{9}$ Es fällt sofort ins Auge, dass dieser Barren (fortan von uns zur einfacheren Unterscheidung auch als ,Londoner Barren“ bezeichnet) weitestgehend dem von uns publizierten (fortan auch ,Degussa Barren“ genannt) entspricht. Auch der hier zur Diskussion stehende ,Londoner Barren“ ist dreifach gestempelt, die Stempel befinden sich in geperltem Rahmen und die beiden äußeren Stempel sind entgegen dem mittleren schwach ausgeprägt und teilweise zerstört bzw. unleserlich. Ebenfalls stimmen die Stempel darin überein, dass es sich bei den beiden äußeren um Bildstempel, bei dem mittleren aber um einen gut lesbaren Textstempel handelt.

Eine Angabe zur genauen Größe von Barren und Stempel des Londoner Exemplars (Länge x Breite x Dicke) fehlt, die Maße dürften aber ungefähr denjenigen des ,Degussa Barrens' (10,6-11 cm x 0,9-2,0 cm x 0,3 cm für den gesamten Barren) entsprechen. Als Gewicht wird für den ,Londoner Barren` 72,2 g angegeben gegenüber 71,2 g beim ,Degussa Barren“ - eine vernachlässigbare Differenz. Einige weitere formale Beobachtungen überraschen. In beiden Fällen finden sich ganz ähnliche Einkerbungen: auf dem Londoner Exemplar einmal eine schräg verlaufende Einkerbung zwischen dem mittleren und dem rechten Stempel und dazu eine nahezu dreiecksförmige auf der Rückseite. Auf dem ,Degussa Barren“ verläuft eine ähnlich schräge Einkerbung unmittelbar durch den rechen Stempel, eine der vorgenannten nicht unähnliche dreiecksförmige auf der Vorderseite quer über den linken Stempel. Trotz unterschiedlichen Sitzes auf den beiden Barren sind die Ähnlichkeiten unverkennbar,

\footnotetext{
${ }^{7}$ Roman Numismatics Limited. Auction IX, 22 march 2015 (London 2015) 220 Nr. 852. Die Kenntnis des Barrens verdanke ich einmal mehr Robert Eberlein, der dem verantwortlichen Leiter des Auktionshauses, R. Beale, unsere Abhandlung zusandte, worauf der Barren aus der Versteigerung herausgenommen wurde. Einige Hinweise zu dem Barren im Auktionskatalog sind allerdings für uns von Belang. R. Beale danke ich für die Zusendung eines aktuellen Fotos des Barrens und für die Erlaubnis zur Veröffentlichung.

${ }^{8}$ Die etwas ungenaue Fundortangabe aufgrund früher Angaben spielt keine Rolle. Einige Funde aus dem Hort von Crasna waren seinerzeit in den Besitz des k. u. k. Gerichtshofes von Kronstadt gelangt.

${ }^{9} \mathrm{Zu}$ diesen Barren s. WIEGELS (Anm. 2) 42 Nr. 1 mit Abb. 5 und 31a sowie 58 Nr. 19 mit Abb. 21 und 31c. Weitere Literatur s. J. P. C. KENT (Hrsg.), in: Coinage and Currency A.D. 300-700. Wealth of the Roman World (London 1977) 535 f. (CM 1894-12-7-1.2); A. M. BuRnETT, Coinage in the Roman World (London 1987) 135; 160 (CM 1894-12-7-1) (nur der vollständige Barren).
} 
was auch die Abbildungen zeigen. Besonders interessant ist ein Vergleich der Metallanalysen. Folgende Werte wurden ermittelt: ${ }^{10}$

London (aus Auktion):
Degussa (Sammlung):
Ag 94,4\%; Cu 5,3; Sn 0,3\%.

Ag 91.5-93,6\%; Cu 6,35-8,5\%; minimale Anteile weiterer Metalle.

Die Werte weichen also leicht, aber mit nur geringen Toleranzen voneinander ab. ${ }^{11}$ In beiden Fällen ist der Kupferanteil vergleichsweise hoch, jedoch konnte weder Blei noch Gold, auch nicht in geringen Spuren, nachgewiesen werden! Wie schon bemerkt, weist dieses darauf hin, dass auch der, Londoner Barren“ nicht antiken Ursprungs ist. Die Indizien deuten vielmehr auf einen gemeinsamen neuzeitlichen Ursprung hin. Daher ist es nicht weiter verwunderlich, wenn beide Barren nahezu zeitgleich das ,Licht der Öffentlichkeit` erblickten.

Was die Stempel als solche betrifft, so handelt es sich bei den beiden Randstempeln um einen bekannten Bildstempel mit den Büsten dreier Kaiser. Deren Identifikation ist umstritten. Mehrheitlich sieht man in ihnen Gratianus, Valentinianus und Theodosius I. (379-383 n.Chr.), im Auktionskatalog werden sie dagegen entsprechend der dort zitierten Literatur als Valentinian I, Valens und Gratian (364-367) identifiziert. Eine eingehende Erörterung des Problems, zu dessen Lösung in der Fachliteratur noch weitere Vorschläge vorgebracht wurden, erübrigt sich an dieser Stelle. ${ }^{12}$ Bei einem Vergleich der Stempel auf dem ,Londoner' und dem ,Degussa Barren“ erkennt man, dass diese nicht nur ähnlich gut bzw. schlecht erhalten sind, sondern auch gleich gerichtet sind, was nicht von vornherein selbstverständlich ist. ${ }^{13}$ Die nach innen gewendeten Texte $\mathrm{NNN}=$ nostri tres und $\mathrm{DDD}=$ domini tres können für beide Bildstempel beider Barren einwandfrei gesichert werden, wobei die ,Verletzungen' des rechten Stempels des ,Degussa Barrens‘ etwas ausgeprägter sind. Dennoch ist die Übereinstimmung in Ausführung und Erhaltung der Bildstempel auf beiden Barren so frappierend, dass eine identische Vorlage zwingend vorauszusetzen ist. ${ }^{14}$ Dies betrifft auch den in beiden Fällen gut erhaltenen Textstempel in der Mitte. Ein genauer und detaillierter Vergleich zeigt, dass beide auf demselben Prägestempel beruhen. Dafür zeugen nicht zuletzt die übereinstimmenden, aber von anderen Textstempeln gleichen Inhalts abweichenden feinen Unterschiede. ${ }^{15}$

\section{Zu lesen ist: LYCIANVS / OBRI • SIC 蛋 (Christogramm)}

SIC steht dabei zweifellos für sig(navit). Bei beiden Exemplaren befindet sich in Z. 2 nur zwischen OBRI und SIC mittig ein Trennpunkt, anders als teilweise in ähnlichen Stempeltexten. Was den Namen Lycianus betrifft, so weichen wir hier von der auch in unserer Abhandlung sowie der gesamten voraufgehenden Literatur weitgehend unhinterfragt übernommenen Lesung Lucianus ab. ${ }^{16}$ Die Unterschiede zwischen dem zwei-

\footnotetext{
${ }^{10}$ Die Werte für den Barren in London wurden dort im Auftrag des Auktionshauses ermittelt und mir von R. Beale zur Verfügung gestellt. - Die Werte des in der ,Sammlung Degussa“ befindlichen Barrens wurden von dieser mehrfach überprüft.

${ }^{11}$ Analysen weiterer Silberbarren - soweit bekannt - haben wir am Ende unserer Studie WIEGELS (Anm. 2) 37 f. zusammengestellt.

${ }^{12}$ Vgl. dazu Wiegels (Anm. 2) 20 mit Anm. 24 und Wiegels (Anm. 4) 39 f. mit Anm. 241-245.

${ }^{13}$ Die Goldbarren aus Crasna zeigen, dass bei Mehrfachstempelung eines Barren häufig ein oder auch mehrere Stempel , auf dem Kopf' stehen. Siehe auch im Folgenden.

${ }^{14} \mathrm{~S}$. dazu auch weiter unten.

${ }^{15}$ An dieser Stelle sei nur auf die Form des gut erkennbaren Christogramms hingewiesen, die von der Ausführung des Symbols auf anderen Barren deutlich abweicht.

${ }^{16}$ Der obere Bereich des zweiten Buchstabens war auf dem ,Degussa Barren` stärker in Mitleidenschaft gezogen gewesen, so dass nur eine senkrechte Haste erkennbar war mit Ansätzen von Serifen im obe-
} 
ten und dem siebten Buchstaben ( $\mathrm{Y}$ bzw. $\mathrm{V}=\mathrm{U}$ ) im Namen sind eindeutig. Wir haben es demnach mit einem griechischen, aber romanisierten Namen zu tun, was zur Grenzsituation zwischen lateinisch-sprachigem und griechisch-sprachigem Raum passt. $^{17}$

Unter historischen Gesichtspunkten gilt das vorrangige Interesse der Klärung der Frage nach Zeitpunkt und möglichem Vorbild für diese ,Fälschungen“. Dabei wollen wir uns an dieser Stelle nicht mit technischen Details möglicher Kopiervorgänge beschäftigen, zumal uns dafür ausreichende Sachkompetenz fehlt. In Erinnerung gebracht sei nur, dass nach Auffinden der Barren von Crasna häufig galvanoplastische Kopien hergestellt wurden, was dazu geführt hat, dass bis heute Unklarheit darüber besteht, wie viele Barren der Fundkomplex von Crasna überhaupt beinhaltet hat. Manche wurden zerstückelt, verkauft, unterschlagen, auf musealer Basis getauscht oder mittels galvanoplastischen Verfahrens vervielfältigt. Wir haben uns in unserer Studie bemüht, wenigstens etwas Klarheit in dieser schwer durchschaubaren Angelegenheit zu schaffen, leider nur mit begrenztem Erfolg. Insbesondere ist es uns nicht gelungen, aus allen diesbezüglich relevanten Museen in Rumänien (Timişoara [früher Temeswar (deutsch) bzw. Temesvár (ungarisch)], Braşov [früher k. u. k. Gerichtshof Kronstadt], vielleicht auch Exemplare im Szekler-Nationalmuseum in Sf. Gheorghe oder in Deva), oder auch in Serbien (Sremska Mitrovica) trotz Bemühens von Kollegen zuverlässige Auskünfte über den Bestand an originalen Barren oder Kopien zu erhalten, und sei es auch nur als negative Anzeige. ${ }^{18}$

Aufgrund der unverkennbaren engen Anlehnung der Stempel auf den hier zur Diskussion stehenden Barren an solche aus dem Fundkomplex von Crasna empfiehlt sich ein etwas genauerer Vergleich. Von den erhaltenen bzw. gut dokumentierten Goldbarren aus Crasna kommt einer unseren Barren auffallend nahe. Dabei handelt es sich um einen heute im Kunsthistorischen Museum in Wien befindlichen Barren mit ebenfalls dreifacher Stempelung: Bildstempel - Textstempel - Bildstempel in gleicher Anordnung, aber mit einem ,kopfstehenden' Bildstempel (hier Abb. 3). ${ }^{19}$ Auch bei diesem Barren weisen die Bildstempel die nach innen gewandten Buchstaben NNN (links) und DDD (rechts) auf, was keineswegs bei allen, wenigstens im Prinzip vergleichbaren Bildstempeln der Fall ist, wo die Verteilung der Buchstaben gerade umgekehrt ist. Bei genauer Betrachtung und Vergrößerung der Abbildung kann auch hier der Name im Textstempel eindeutig als Lycianus (statt Lucianus) gelesen werden. Der ,Wiener Barren' befand sich lange in privatem Besitz und wurde dann 1903 von dem genannten Museum zusammen mit einem Nachlass erworben. Ob hier zwischen Auffinden - privatem Erwerb - Museumsakzise und den modernen Kopien in Silber ein Bezug herzustellen ist, lässt sich nicht sagen; jedenfalls müsste derartiges in der Zeit zwischen Auffinden und Erwerb durch das Museum in Wien ins Werk gesetzt worden sein. Um eine einfache Kopie der gesamten Stempelfolge kann es sich jedenfalls nicht

ren Bereich, die wir jetzt aber als zugehörig zu einem Buchstaben „Y“ deuten können. Dasselbe lässt sich auch bei anderen vergleichbaren Stempeln ausmachen, nicht zuletzt bei dem mittleren Stempel des Barrens in der Londoner Auktion. - Gewisse Bedenken gegen die ältere Lesung des Namens wurden von uns bereits in WIEGELS (Anm. 2) 21 mit Anm. 25 geäußert, ohne allerdings daraus weitere Konsequenzen zu ziehen. Dass „, $\mathrm{Y}^{\prime}$ und ,V $=\mathrm{U}$ “ bei Stempeln zuweilen kaum unterscheidbar sind, ist hinreichend bekannt.

${ }^{17}$ Der Name $\Lambda$ ${ }^{10}{ }^{10}$ (Lykios) ist im Griechischen häufig belegt.

${ }^{18}$ Dieses gravamen betrifft nicht die großen Museen wie Budapest, Bukarest, London, Paris oder Wien, denen wir in verschiedener Hinsicht zu Dank verpflichtet sind. - Die diesbezügliche Forschungsliteratur ist alles andere als eindeutig und in manchen Fällen eher für Unklarheiten verantwortlich.

${ }^{19}$ Unter den Goldbarren aus Crasna ist diese Weise der Stempelung bislang singulär; vgl. dazu WIEGELS (Anm. 2) 59 Nr. 20: „Serie IV“. 
handeln. Noch ein Detail gilt es in diesem Zusammenhang zu bedenken. Bei aller sachlichen und formalen Übereinstimmung zwischen dem ,Degussa-Barren' und dem ,Londoner Barren' ist unverkennbar, dass die Platzierung der drei Stempel auf den Barren leicht voneinander abweicht. Beim ,Degussa-Barren“ ist der Abstand vom linken zum mittleren Stempel etwas größer als derjenige zum rechten Randstempel, beim ,Londoner-Barren` verhält es sich genau umgekehrt. ${ }^{20}$ Die Stempelungen beruhen also nicht auf einer ganzheitlichen, alle drei Stempel gleichermaßen erfassenden Vorlage (Patrize), sondern um verschiedene Einzelstempel! ${ }^{21}$ Eine Antwort auf die Frage, ob es einen den beiden Silberbarren entsprechenden, dreifach und gleichgerichtet gestempelten originalen Goldbarren gab, muss offen bleiben. Der Goldbarren in Wien mag auch lediglich als Vorbild gedient haben. Aber es würde kaum überraschen, wenn weitere Exemplare gestempelter Silberbarren auftauchen würden.

Zugegeben: In historischer Perspektive ist der negative Befund zu den Silberbarren, die in der Weise von Goldbarren gestempelt wurden, am wichtigsten, einige Randnotizen ausgenommen. Leider bleibt die Frage nach Umfang und ursprünglicher Bestimmung der Goldbarren von Crasna ebenso unbeantwortet wie diejenige zu den Funden von Goldbarren aus dem unweit gelegenen Feldioara. Doch diese stehen hier erst gar nicht zur Debatte.

\footnotetext{
${ }^{20}$ Übrigens ist der Textstempel bei dem Goldbarren in Wien auch nicht exakt mittig platziert.

${ }^{21}$ Die Bildstempel könnten allerdings auch auf ein und demselben Prägestempel beruhen.
} 
Frankfurter elektronische Rundschau zur Altertumskunde 27 (2015)

\section{Abbildungen}

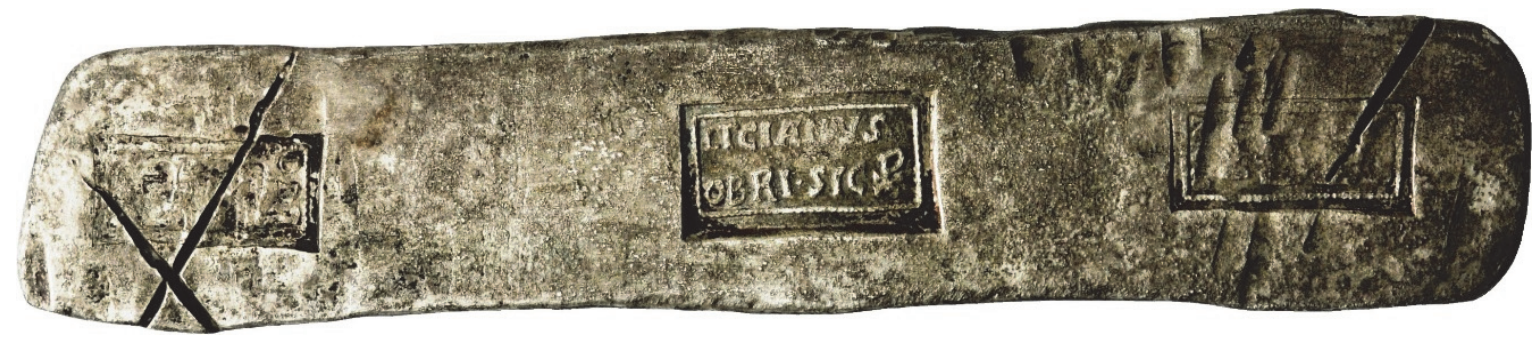

Abb. 1: Silberbarren mit Stempeln. Vor- und Rückseite. - AO: Sammlung Degussa.

Foto: Degussa Goldhandel GmbH, München.
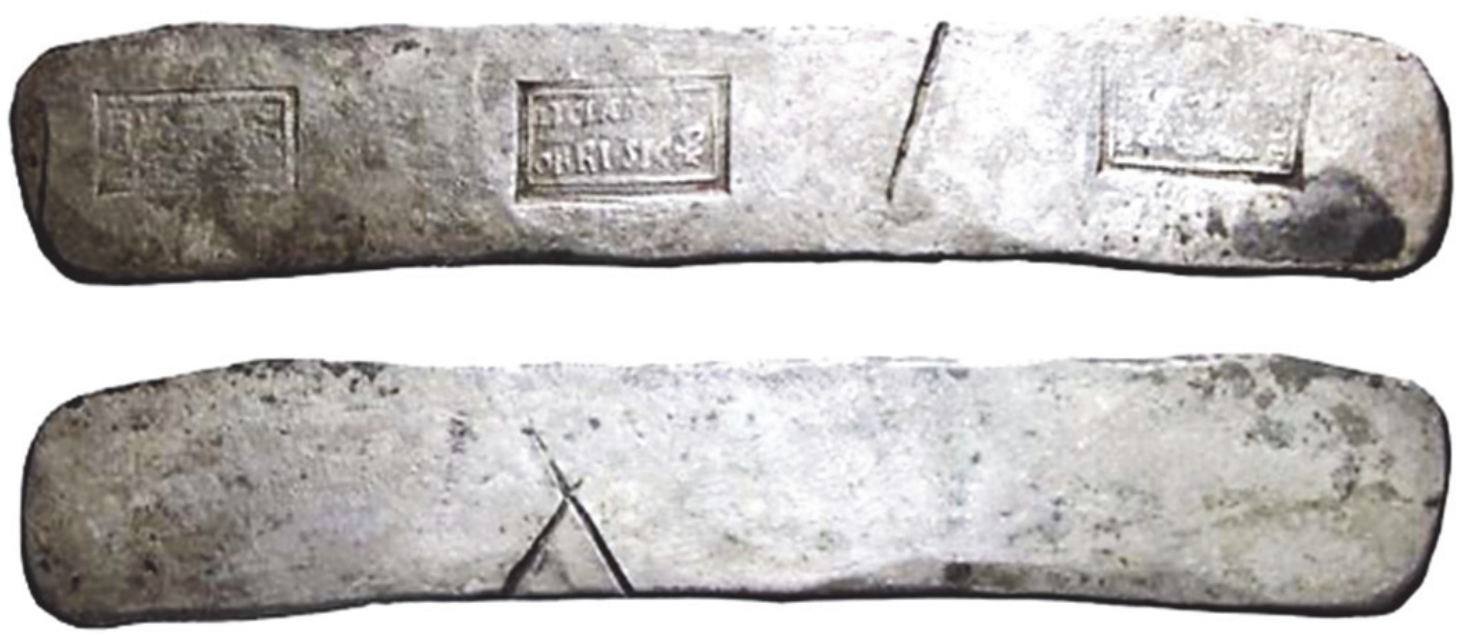

Abb. 2: Silberbarren mit Stempeln. Vor- und Rückseite. - AO: London. Foto: Roma Numismatic Limited, London. 
Frankfurter elektronische Rundschau zur Altertumskunde 27 (2015)

Abb. 3: Crasna (Rumänien): Dreifach gestempelter spätantiker Goldbarren. AO: Wien, Kunsthist. Museum. Foto: Österreich. Nationalbank-Dateien. 